\title{
Dejal@Bot: Un chatbot aplicable en el tratamiento de la deshabituación tabáquica
}

\author{
José Francisco Ávila-Tomás 1, Eduardo Olano-Espinosa 2,*, César Minué-Lorenzo ${ }^{3}$, Francisco \\ Javier Martínez-Suberbiola ${ }^{4}$, Blanca Matilla-Pardo ${ }^{5}$, María Encarnación Serrano-Serrano ${ }^{6}$, María \\ Victoria Güeto-Rubio ${ }^{7}$ y Grupo Dej@lo \\ 1 Atención Primaria de Salud, Servicio Madrileño de Salud; Estudiante de doctorado, Departamento de \\ Medicina Preventiva y Salud Pública, Universidad Rey Juan Carlos; Grupo de Trabajo de Innovación \\ Tecnológica y Sistemas de Información de semFYC; https://orcid.org/0000-0003-0754-744X \\ 2 Atención Primaria de Salud, Servicio Madrileño de Salud; Grupo de Abordaje del Tabaquismo (GAT) \\ SoMaMFyC; https://orcid.org/0000-0001-5031-6298 \\ 3 Atención Primaria de Salud, Servicio Madrileño de Salud; Grupo de Abordaje del Tabaquismo (GAT) \\ SoMaMFyC; https://orcid.org/0000-0002-8015-3378 \\ 4 Atención Primaria de Salud, Servicio Madrileño de Salud; https://orcid.org/0000-0001-7019-0688 \\ 5 Atención Primaria de Salud, Servicio Madrileño de Salud; https://orcid.org/0000-0001-6379-3608 \\ 6 Atención Primaria de Salud, Servicio Madrileño de Salud; Grupo de Abordaje del Tabaquismo (GAT) \\ SoMaMFyC; Departamento de Medicina y Especialidades Médicas, Universidad de Alcalá; \\ https://orcid.org/0000-0003-3150-2348 \\ 7 Atención Primaria de Salud, Servicio Navarro de Salud; \\ * Autor correspondencia: e.oeoeoeoe@gmail.com \\ DOI: https://doi.org/10.37536/RIECS.2020.5.1.196
}

Recibido: 16/03/2020; Aceptado: 18/04/2020; Publicado: 30/05/2020

Resumen: En el contexto del estudio "Efectividad de un bot conversacional para dejar de fumar en población adulta: ensayo clínico pragmático en Atención Primaria (Déj@lo)" (PI17/01942) que evalúa la efectividad de una intervención para ayudar a los fumadores a abandonar el tabaco a través de un bot conversacional para teléfonos inteligentes en comparación con el manejo habitual en Atención Primaria de Salud debíamos construir un chatbot sobre el que no existen aún unos estándares de calidad y nos basamos en los existentes para aplicaciones. Un bot (o chatbot) es una aplicación informática dotada de inteligencia artificial (IA) capaz de generar una conversación bidireccional entre un ser humano y una máquina (robot) a través de un interfaz conversacional o chat. En este trabajo presentamos la herramienta utilizada y los criterios que hemos seguido para su construcción y elaboración de contenidos.

Palabras Clave: Chatbot, Inteligencia artificial, Tabaco, Deshabituación tabáquica.

Abstract: In the context of the study "Effectiveness of a conversational bot to quit smoking in the adult population: pragmatic clinical trial in Primary Care (Déj@lo)" (PI17/01942) that evaluates the effectiveness of an intervention to help smokers to quit tobacco through a conversational bot for smartphones compared to the usual management in primary health care we had to build a chatbot on which there are no quality standards yet and we rely on existing standards for applications. A bot (or chatbot) is a computer application with artificial intelligence (AI) capable of generating a two-way conversation between a human being and a machine (robot) through a conversational interface or chat. In this work we present the tool used and the criteria we have followed for its construction.

Key words: Chatbot, Artificial intelligence, Tobacco, Smoking cessation. 


\section{Introducción}

Actualmente existen numerosos ensayos clínicos que exploran la utilización de herramientas digitales en procesos sanitarios. En relación al tabaquismo se han desarrollado y evaluado diversas apps [1] pero pocos chatbots para la atención a fumadores [2] a pesar de que la eficacia de intervenciones para dejar de fumar con telemedicina parece ofrecer resultados similares a procesos presenciales [3]. En otros aspectos de la prevención y promoción de salud sí existen ensayos clínicos con bots como en consumo de alcohol y drogas en adolescentes [4] u obesidad en pediatría [5].

A priori pudiera parecer que el uso de dispositivos electrónicos va dirigido a jóvenes y adolescentes por su mejor manejo tecnológico, sin embargo, existen también evaluaciones en adultos y ancianos [6]. Una variable interesante sería cuantificar la aceptación por parte de usuarios [7] y profesionales [8] de estas herramientas.

La implementación de la tecnología de bots en dispositivos móviles tiene varias ventajas frente a las apps:

1. Un chatbot no es un programa para instalar por lo que respeta más la privacidad del usuario.

2. Las apps suelen basar su modelo de negocio en publicidad dirigida lo que requiere acceso a datos personales.

3. Las apps tienen un funcionamiento lineal a través de botones de forma rígida y no adaptable al usuario.

4. El uso de chatbots plantea pocas dificultades ya que funciona a través de apps de mensajería, las más extendidas y utilizadas entre los usuarios de móviles.

Se pueden establecer unos criterios de calidad para apps orientadas al tratamiento del tabaquismo que surgen de estudios observacionales tanto cuantitativos como cualitativos.

El requisito más valorado por parte de los profesionales es que sus contenidos correspondan con la evidencia científica [9] y que ofrezcan seguridad y privacidad. Otras características valoradas son: coste, adaptabilidad al paciente y a su situación, ayuda al reconocimiento y abordaje de recaídas, información sobre fármacos y efectos secundarios, seguimiento, apoyo social y comunicación con profesionales sanitarios [10].

Las características más valoradas por parte de los usuarios [9] son: herramienta altamente personalizada, de bajo coste, que ayude a afrontar síntomas de abstinencia y efectos secundarios de fármacos y que rastree su progreso (días sin fumar, dinero ahorrado o beneficios para la salud). El ajuste a la evidencia científica o el aval por expertos o sociedades científicas no es determinante.

\section{Desarrollo de la herramienta}

En nuestro caso se creó un grupo multidisciplinar con generación de un lenguaje común para compaginar elementos narrativos (contenidos científicos) con la estructura de programación del chatbot. En este grupo participamos tres médicos de familia, dos de ellos expertos en tabaquismo y un tercero experto en aplicaciones tecnológicas sanitarias junto a dos programadores, uno de ellos filósofo con experiencia en filosofía de la ciencia.

En una revisión previa de la bibliografía comprobamos la existencia de ensayos de deshabituación con mensajes SMS y con algunas apps. Ninguna de las apps existentes en el mercado se adaptaba a nuestras necesidades y fuimos conscientes de las ventajas de un chatbot, por lo que nos propusimos construir una nueva herramienta.

Los aspectos que determinaron la construcción de nuestro chatbot se resumen en la Tabla I. 
Tabla I Aspectos que determinaron la construcción de nuestro chatbot

\begin{tabular}{|c|c|c|c|}
\hline & Participantes & Tareas/Requisitos & Soluciones dadas \\
\hline $\begin{array}{l}\text { Adecuación a la } \\
\text { evidencia científica }\end{array}$ & $\begin{array}{l}\text { Médicos } \\
\text { expertos en } \\
\text { tabaquismo }\end{array}$ & $\begin{array}{l}\text { Búsqueda y selección de } \\
\text { Guías de Práctica Clínica } \\
\text { (GPC) y metaanálisis de } \\
\text { referencia en tabaquismo }\end{array}$ & $\begin{array}{l}\text { Todas las recomendaciones } \\
\text { clínicas del bot de basan en } \\
\text { GPC. }\end{array}$ \\
\hline Seguridad de datos & Programadores & $\begin{array}{c}\text { Estudio de las diferentes } \\
\text { plataformas que soportan } \\
\text { chatbots }\end{array}$ & $\begin{array}{c}\text { La información va cifrada } \\
\text { punto a punto. } \\
\text { No hay acceso a número de } \\
\text { teléfono. } \\
\text { El servidor donde } \\
\text { almacenamos los datos del bot } \\
\text { cumple con todos los } \\
\text { requisitos de seguridad y } \\
\text { privacidad. }\end{array}$ \\
\hline $\begin{array}{c}\text { Interacción con el } \\
\text { usuario }\end{array}$ & $\begin{array}{c}\text { Médicos / } \\
\text { Programadores }\end{array}$ & $\begin{array}{l}\text { El diálogo con el bot es } \\
\text { bidireccional y puede } \\
\text { iniciarse por el humano o } \\
\text { por la máquina utilizando } \\
\text { siempre un lenguaje natural } \\
\text { en la comunicación }\end{array}$ & $\begin{array}{c}\text { El bot se adapta a las } \\
\text { necesidades de información y } \\
\text { tono de ánimo por el que } \\
\text { atraviesa el usuario en el } \\
\text { proceso de abandono del } \\
\text { tabaco. }\end{array}$ \\
\hline Multimedia & $\begin{array}{c}\text { Médicos / } \\
\text { Programadores }\end{array}$ & $\begin{array}{c}\text { Nos permite enviar } \\
\text { elementos multimedia } \\
\text { generando una } \\
\text { comunicación personalizada } \\
\text { donde el usuario puede } \\
\text { decidir el formato en que } \\
\text { recibe la información } \\
\text { solicitada }\end{array}$ & $\begin{array}{c}\text { Hemos desarrollado } 30 \\
\text { píldoras de información } \\
\text { multimedia que abarcan } \\
\text { temas desde el tratamiento } \\
\text { médico específico (en caso que } \\
\text { haya sido prescrito), estudio } \\
\text { de mitos asociados al } \\
\text { abandono del tabaco, } \\
\text { funcionamiento de la curva } \\
\text { del deseo, manejo de las } \\
\text { desautomatizaciones, } \\
\text { prevención de recaídas, } \\
\text { ejercicios de respiración o } \\
\text { manejo de la ansiedad. }\end{array}$ \\
\hline Gamificación & $\begin{array}{c}\text { Médicos / } \\
\text { Programadores }\end{array}$ & $\begin{array}{l}\text { Mecánica de juego a través } \\
\text { de niveles que se alcanzan } \\
\text { cuando el usuario adquiere } \\
\text { conocimientos o habilidades } \\
\text { a lo largo del proceso. Se } \\
\text { premia su implicación en la } \\
\text { actividad otorgándole un } \\
\text { nivel o estatus distintivo del } \\
\text { resto de jugadores y anima a } \\
\text { los usuarios inferiores a } \\
\text { igualarle. }\end{array}$ & $\begin{array}{c}\text { Se estableció mediante la } \\
\text { entrega de insignias cuando el } \\
\text { usuario alcanza un objetivo } \\
\text { previamente conocido } \\
\text { (días/semanas sin fumar) o } \\
\text { elabora una tarea importante } \\
\text { en el proceso (lista de motivos } \\
\text { o selección del día D). }\end{array}$ \\
\hline
\end{tabular}


El proceso de elaboración de la herramienta tuvo tres etapas:

1. Desarrollo del esqueleto lógico. En una línea temporal desde el día -15 hasta un año después del abandono del tabaco se generaron todas las posibilidades de contacto entre el fumador y el chatbot en un árbol de opciones que sirvió de esqueleto para su programación.

2. Desarrollo de un guion. Cada uno de estos contactos se guionizó como si fuera una obra de teatro. Con este planteamiento de escenarios se ajustó el desarrollo del esqueleto lógico ya que aparecieron situaciones no previstas inicialmente. El guion se complementó con campos semánticos y diccionarios que iniciaron el aprendizaje del chatbot que identifica, de forma progresiva y cada vez con más acierto, las necesidades expresadas por el usuario.

3. Prueba: Durante un mes se dio acceso al chatbot a un grupo de expertos en tabaquismo y tecnología que utilizaron la herramienta para detectar fallos de conexión de escenarios, disfunciones lógicas y evaluar los contenidos. Se realizaron los últimos ajustes tanto en el esqueleto como en el guion y los materiales.

Humanizar la tecnología es una necesidad ya que esta ha de estar al servicio del ser humano y no al servicio de sus productos. El proceso de dejar de fumar es una decisión personal que afecta a nuestro plano personal o íntimo y tener como interlocutor una máquina podría significar una relación fría y poco empática. Por esta razón, queríamos humanizar nuestro robot en varios aspectos:

1. Imagen humanoide: En la cultura, sobre todo cinematográfica del siglo $\mathrm{XX}$, tenemos ejemplos de humanización robótica física o emocional. Desde comportamientos agresivos (Terminator 1984 o Robocop -1987), conflictos humanos (Blade Runner -1982), aspecto infantil (Wall-e -2008) o robots que interactúan entre ellos como C3PO y R2D2 (Stars Wars). Deseábamos una imagen de un robot cercano a las pasiones humanas sin dejar de tener un aspecto de robot y descubrimos en nuestra búsqueda a Bender B. Rodríguez, robot de la serie Futurama (FOX 1999-2013). En Futurama, los robots representan seres sin limitaciones éticas, pero formando parte plena de la sociedad. Utilizando un elemento «poco familiar» (robots) se nos muestra «algo familiar» (comportamiento humano). Buscamos imágenes sin derechos de autor para poder utilizarlas en nuestro proyecto y a través de diferentes modelos vectoriales libres generamos a nuestro Dejal@Bot. Le "dotamos de sentimientos humanos" a través de emoticonos que representan estados de ánimo que se han establecido como estándares en su significado de comunicación. (Fig. 1)

2. Estructuras conversacionales: Se elaboraron colecciones de palabras o frases con los que un fumador podía expresar síntomas, situaciones o dudas. Se generaron diccionarios de miedos y preocupaciones para dejar de fumar, de síntomas asociados al consumo de tabaco, motivos para dejar de fumar, diccionario de recaídas y diccionario de fantasía de control. Se generó también un diccionario de "insultos, tacos o palabras malsonantes" para que el bot reaccionara a estas posibles expresiones. De esta manera el bot va aprendiendo un lenguaje natural y se adapta y reacciona en la interacción con el humano.

3. Adaptación a los procesos psicológicos. El tipo de lenguaje del chatbot, la estructura semántica y el tono van cambiando según las situaciones que plantea el usuario. Por ejemplo, después del día $\mathrm{D}$ se pregunta al inicio de cada interacción si se sigue fumando; ante una respuesta negativa el tono es lúdico y se ofrece una recompensa por la abstinencia, mientras que si la respuesta es positiva o se detecta una recaída el tono conversacional es de ayuda y de empatía. 


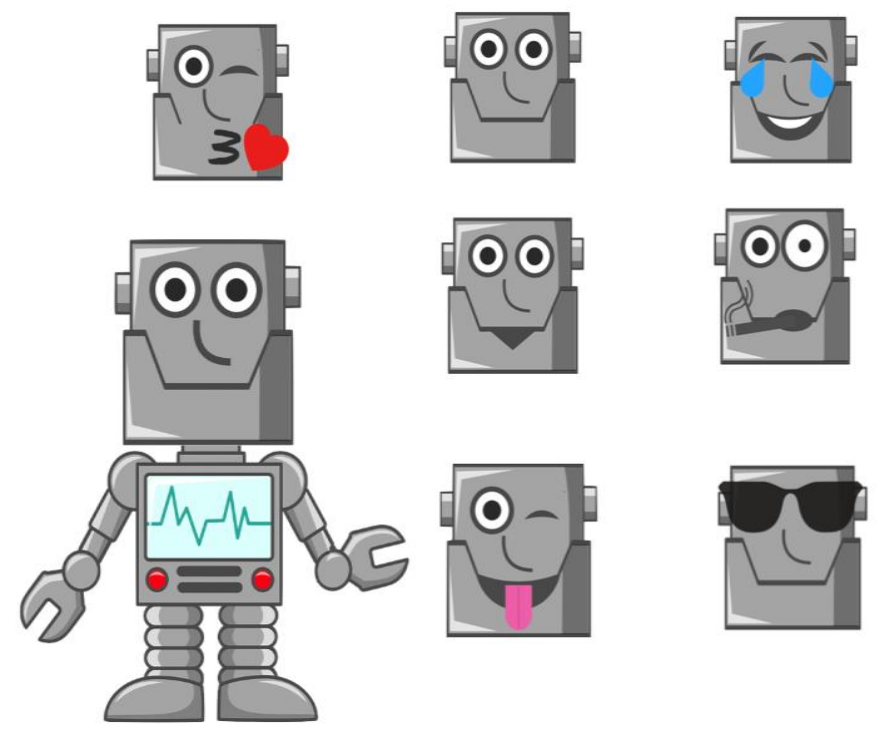

Figura 1 Diferentes aspectos de Dej@loBot utilizando emoticonos faciales

\section{Conclusiones}

1. Necesidad de evaluar herramientas digitales. Cada vez vamos a tener más herramientas digitales de apoyo al diagnóstico o tratamiento de procesos sanitarios que deberían ser evaluadas a través de ensayos clínicos que demostraran su validez.

2. Grupos multidisciplinares. La generación de lenguajes comunes en un entorno que trasciende a los profesionales sanitarios es una necesidad actual.

3. Equipos de trabajo que tengan en cuenta las valoraciones previas de usuarios y profesionales antes de iniciar el proceso de construcción de una herramienta.

Agradecimientos: Agradecer el apoyo metodológico en el ensayo clínico de la Fundación para la Investigación e Innovación en Atención Primaria (FIIBAP). Agradecer a Joaquín Herrero Pintado, uno de los programadores del bot, la lectura del artículo y sus correcciones. El equipo DejaloBot está formado por profesionales sanitarios de diferentes centros de salud del Servicio Madrileño de Salud citados en el material complementario 1.

Contribución de los autores: Todos los autores firmantes han contribuido de manera sustancial al desarrollo y revisión del artículo. JFAT, EOE y CML han redactado el artículo y han formado parte integrante en el equipo del desarrollo del bot. FJMS, BMP, MESS han contribuido en la concepción y diseño del ensayo clínico sobre el que se basa este artículo y han revisado su redacción y corrección. MVGR ha revisado el manuscrito inicial y ha aportado aspectos esenciales en su concepción y desarrollo. Todos los autores han leído, corregido y aprobado el manuscrito previo a su remisión a esta revista. Los investigadores del Grupo Dej@lo son los profesionales que están realizando el trabajo de campo del ensayo clínico.

Financiación: Este artículo se basa en un ensayo clínico financiado por el Fondo de Investigaciones Sanitarias ISCIII y el European Regional Development Fund (ERDF) con número de registro: PI17 / 01942. Los financiadores no han desempeñado papel en el diseño del estudio, la recogida de datos ni en la elaboración de conclusiones. El organismo financiador ha realizado una revisión por pares del protocolo del ensayo clínico.

Conflictos de Intereses: El investigador principal EOE ha recibido una subvención de la FIIBAP en la convocatoria de subvenciones 2018 para la promoción de programas de investigación. El resto de autores declaran no tener conflictos de interés. 


\section{Referencias Bibliográficas}

1. Nomura A, Tateno H, Masaki K, Muto T, Suzuki S, Satake K, et al. A Novel Smoking Cessation Smartphone App Integrated With a Mobile Carbon Monoxide Checker for Smoking Cessation Treatment: Protocol for a Randomized Controlled Trial. JMIR Res Protoc. 2019;8(2):e12252. [Texto Completo]

2. Perski O, Crane D, Beard E, Brown J. Does the addition of a supportive chatbot promote user engagement with a smoking cessation app? An experimental study. Digital Health. 2019; 5:205520761988067. [Texto Completo]

3. Nomura A, Tanigawa T, Muto T, Oga T, Fukushima Y, Kiyosue A, et al. Clinical Efficacy of Telemedicine Compared to Face-to-Face Clinic Visits for Smoking Cessation: Multicenter Open-Label Randomized Controlled Noninferiority Trial. J Med Internet Res.2019;21(4): e13520. [Texto Completo]

4. Crutzen R, Peters G-JY, Portugal SD, Fisser EM, Grolleman JJ. An artificially intelligent chat agent that answers adolescents' questions related to sex, drugs, and alcohol: an exploratory study. J Adolesc Health.2011;48(5):514-9.

5. Stephens TN, Joerin A, Rauws M, Werk LN. Feasibility of pediatric obesity and prediabetes treatment support through Tess, the AI behavioral coaching chatbot. Transl Behav Med. 2019;9(3):440-7.

6. Piau A, Crissey R, Brechemier D, Balardy L, Nourhashemi F. A smartphone Chatbot application to optimize monitoring of older patients with cancer. Int J Med Inform. 2019; 128:18-23.

7. Morris RR, Kouddous K, Kshirsagar R, Schueller SM. Towards an Artificially Empathic Conversational Agent for Mental Health Applications: System Design and User Perceptions. J Med Internet Res. 2018;20(6):e10148. [Texto Completo]

8. Palanica A, Flaschner P, Thommandram A, Li M, Fossat Y. Physicians' Perceptions of Chatbots in Health Care: Cross-Sectional Web-Based Survey. J Med Internet Res. 2019;21(4): e12887. [Texto Completo]

9. McClure JB, Hartzler AL, Catz SL. Design Considerations for Smoking Cessation Apps: Feedback From Nicotine Dependence Treatment Providers and Smokers. JMIR mHealth uHealth. 2016;4(1): e17. [Texto Completo]

10. Bhattacharya A, Vilardaga R, Kientz JA, Munson SA. Lessons from Practice: Designing Tools to Facilitate Individualized Support for Quitting Smoking. En: Proceedings of the $2017 \mathrm{CHI}$ Conference on Human Factors in Computing Systems [Internet]. New York, NY, USA: ACM; 2017. p. 3057-3070. (CHI '17). [Texto Completo]

(C) 2020 por los autores; Esta obra está sujeta a la licencia de Reconocimiento 4.0 Internacional de Creative Commons. Para ver una copia de esta licencia, visite http://creativecommons.org/licenses/by-nc-nd/4.0/. 